\title{
Posterior inferior cerebellar artery reimplantation: buffer lengths, perforator anatomy, and technical limitations
}

\author{
Ali Tayebi Meybodi, MD, Michael T. Lawton, MD, Xuequan Feng, MD, PhD, and Arnau Benet, MD \\ Skull Base and Cerebrovascular Laboratory, Department of Neurological Surgery, University of California, San Francisco, \\ California
}

OBJECTIVE Reimplantation of the posterior inferior cerebellar artery (PICA) to the vertebral artery (VA) is a safe and effective bypass option after deliberate PICA sacrifice during the treatment of nonsaccular and dissecting aneurysms at this location. However, the anatomy and limitations of this technique have not been studied. The goal of this study was to define the surgical anatomy and buffer lengths specific to the proximal segment of the PICA related to 2 variations of PICA reimplantation: 1) reimplantation "along-VA" (simulating a dissecting VA aneurysm), and 2) reimplantation "acrossVA" (simulating a nonclippable, proximal PICA aneurysm).

METHODS Ten cadaver heads (20 sides) were prepared for surgical simulation. Twenty far-lateral approaches were performed. The PICA was mobilized and reimplanted onto the VA according to 2 different paradigms: 1) transposition along the axis of the VA (along-VA) to simulate a dissecting VA, and 2) transposition perpendicular to the axis of the VA (across-VA) to simulate a nonclippable, proximal PICA aneurysm. The buffer lengths provided by mobilization of the artery in each paradigm were measured and the anatomy of perforator branching on the proximal PICAs was analyzed.

RESULTS The PICA was reimplanted in all surgical simulations. The most common perforating artery on the $P_{1}$ and $P_{2}$ segments was the short circumflex type. No direct perforator was found on the $P_{1}$ segment. The mean buffer length with reimplantation along the VA axis was $13.43 \pm 4.61 \mathrm{~mm}$, and it was $6.97 \pm 4.04 \mathrm{~mm}$ with reimplantation across the VA. The PICA was less maneuverable when it was reimplanted across the VA, due to perforator branches of the PICA $\left(P_{3}\right.$ segment).

CONCLUSIONS The buffer lengths measured in this study describe the limitations of PICA reimplantation as a revascularization procedure for nonsaccular aneurysms in this location. PICA reimplantation is a revascularization option for dissecting VA aneurysms incorporating the PICA origin that are $<13 \mathrm{~mm}$ in length, and for nonsaccular proximal PICA aneurysms that are $<6 \mathrm{~mm}$ in diameter. The final decision to reimplant the PICA depends on careful inspection of perforator anatomy that is not visible preoperatively on angiography, as well as an assessment of technical difficulty intraoperatively.

http://thejns.org/doi/abs/10.3171/2015.8.JNS151411

KEY WORDS reimplantation; posterior inferior cerebellar artery; far-lateral craniotomy; dissecting aneurysm; vertebral artery; vascular disorders

A NEURYSMS affecting the vertebral artery (VA) and posterior inferior cerebellar artery (PICA) can be challenging, especially when the aneurysm is nonsaccular, the PICA originates from the aneurysm base, or the parent artery is dissected. Repair of these aneurysms often sacrifices the PICA, in which case a bypass might be indicated and PICA reimplantation might be a consideration. $5,7,11,12,17,19,25$ Successful PICA reimplantation depends on the anatomy of the recipient artery, specifically the presence of medullary perforators on the proximal $\mathrm{P}_{1}$ segment that might tether the PICA, and the extra length proximally that allows the PICA to be transposed (buffer length). Although reimplantation of the PICA to the VA has been previously reported $5,10,12,15,19,23$ and included in our own clinical series of 35 intracranial-to-intracranial bypasses for PICA aneurysms, the anatomy and limitations of this procedure have not been studied. Variables such as number, size, and type of the PICA perforators, as well

ABBREVIATIONS PICA = posterior inferior cerebellar artery; VA = vertebral artery.

SUBMITTED June 17, 2015. ACCEPTED August 26, 2015.

INCLUDE WHEN CITING Published online January 22, 2016; DOI: 10.3171/2015.8.JNS151411. 
as buffer lengths, are critically important. However, they cannot be easily visualized on preoperative angiography and therefore have not been quantified. Morphometric analysis in cadaveric specimens is needed to appreciate these factors. ${ }^{1,2,7,12,14,17,18,24}$

In this study, we sought to define the specific proximal PICA anatomy and buffer lengths related to 2 variations of PICA reimplantation: 1) reimplantation "along-VA" (simulating a dissecting VA aneurysm), and 2) reimplantation "across-VA" (simulating a nonclippable, proximal PICA aneurysm). We performed surgical simulations via a farlateral approach using cadavers to maximize the surgical applicability of our findings.

\section{Methods}

Ten cadaver heads without cranial disease were prepared for surgical simulation using our customized formula. ${ }^{4}$ Twenty classic far-lateral approaches using a hockey-stick skin incision were completed to access the $\mathrm{V}_{4}$ segment and the VA-PICA junction. The posterior third of the occipital condyle was removed to facilitate access to the lateral gutter of the cerebellomedullary region. After opening the dura, the first 2 segments of the PICA (anterior medullary $\left[\mathrm{P}_{1}\right]$ and lateral medullary $\left[\mathrm{P}_{2}\right]^{20}$ were examined. The lengths of each segment, as well as the number and type of perforating branches, were recorded. Perforators were described according to the nomenclature proposed by Lister et al.:18 direct, short circumflex, and long circumflex perforators. However, to avoid ambiguity and errors in classification, a perforating artery was labeled as direct only if the parent artery lay on the brainstem surface, with the perforator branch directly entering the brainstem at the same level. Otherwise, it was categorized as a short circumflex perforator, unless the course of the artery included a $>90^{\circ}$ turn around the brainstem, in which case it would be considered as long circumflex.

Next, the origin of the PICA was cut from the VA and mobilized in 2 different fashions relative to the VA. To recreate the surgical scenario in which the PICA originates from a fusiform VA aneurysm but the artery itself is intact, the PICA was mobilized as far along the VA axis as possible (either down or up, relative to the origin of the PICA on the VA). This allowed measurement of the length of the VA that could be sacrificed and still allow the PICA to be transposed and reimplanted (along-VA paradigm; Fig. 1A). In the across-VA paradigm, it was assumed that the proximal PICA was aneurysmal and needed to be excised, whereas the VA was considered disease free. Thus, the PICA was pulled perpendicularly across the axis of the VA to measure the buffer length of the PICA available for excision, while still allowing reimplantation (Fig. 1B). Care was taken to prevent undue tension to the perforating branches and the PICA while mobilizing the artery. Length measurements were taken using a stereotactic system (Stryker), as defined by our team. ${ }^{3}$ The Pearson correlation coefficient was calculated to assess the statistical correlation between the lengths of $\mathrm{P}_{1}$ and $\mathrm{P}_{2}$ segments and the buffer length provided in each paradigm. The Student's t-test was used to compare the means of buffer lengths among different groups of branching configuration of perforators.

\section{Results}

Reimplantation of the PICA was successfully performed

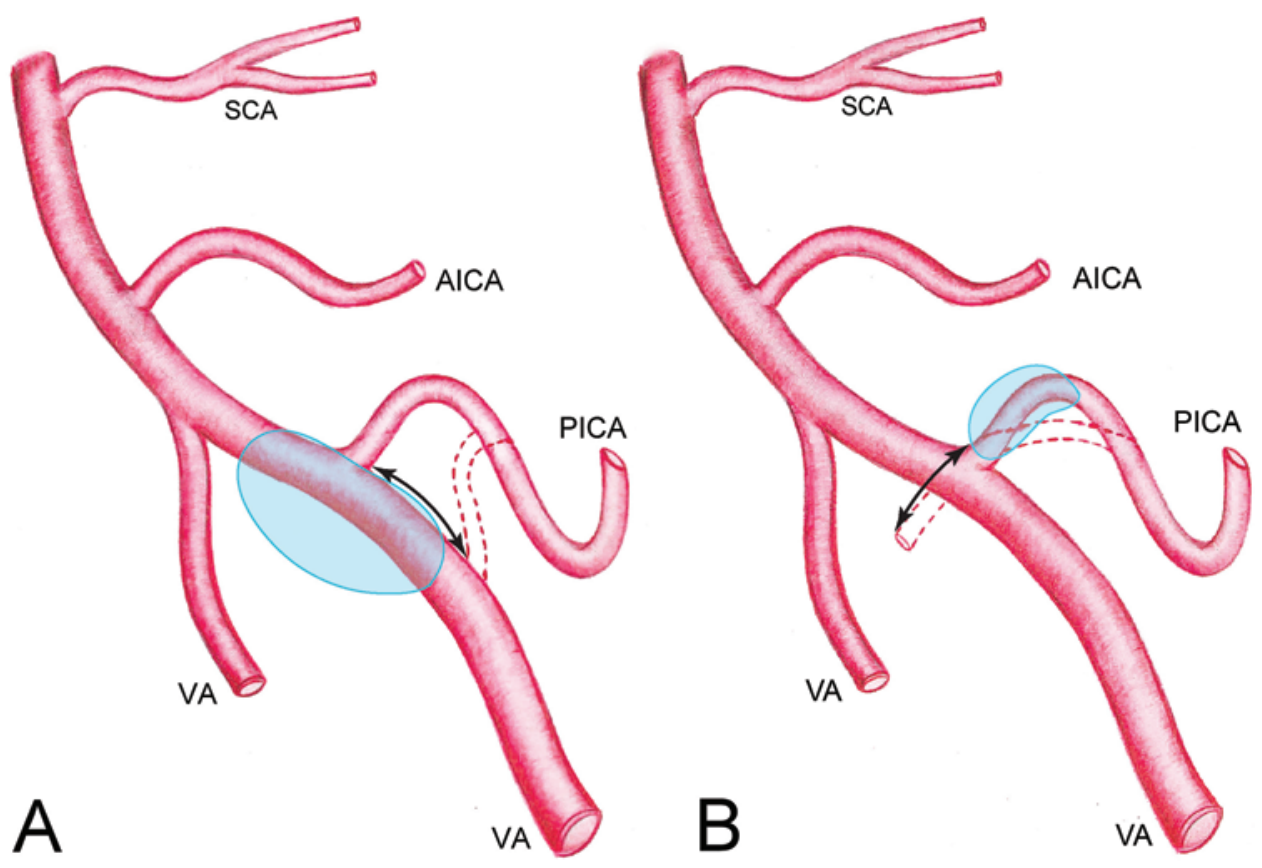

FIG. 1. A schematic drawing showing the 2 different paradigms and buffer lengths analyzed in the present study. A: Along-VA reanastomosis. B: Across-VA reanastomosis. The blue areas represent the aneurysmal part of the respective artery. Double arrows show the mobilization paradigm of the PICA. AICA = anterior inferior cerebellar artery; SCA = superior cerebellar artery. Printed with permission from the Skull Base and Cerebrovascular Laboratory, University of California, San Francisco. Copyright Arnau Benet. Published with permission. Figure is available in color online only. 
in all specimens (20 PICAs; Figs. 2 and 3). Two hypoplastic PICAs and 1 extradural PICA (originating from the $\mathrm{V}_{2}$ segment of the VA) were excluded from the statistical analysis. The number and type of perforating arteries from the $\mathrm{P}_{1}$ and $\mathrm{P}_{2}$ segments, the lengths of these 2 segments, and buffer length measurements performed under 2 different paradigms (along-VA vs across-VA mobilization) are reported in Table 1. The most common perforator type in both the $\mathrm{P}_{1}$ and $\mathrm{P}_{2}$ segments was short circumflex. There were no direct perforators in the $\mathrm{P}_{1}$ segments. In 1 PICA, a short circumflex perforator originated $3 \mathrm{~mm}$ from the origin of the artery, which precluded across-VA mobilization. In 4 other cases, the first perforator origination pattern allowed $\leq 3$ $\mathrm{mm}$ of buffer length. These cases were collectively named as early branching pattern of perforators. Early branching did not significantly affect the buffer length in the alongVA paradigm (i.e., average buffer length provided by alongVA mobilization in PICAs with early branching vs PICAs without early branching; $p>0.05$ ).

The average buffer length provided by mobilization of

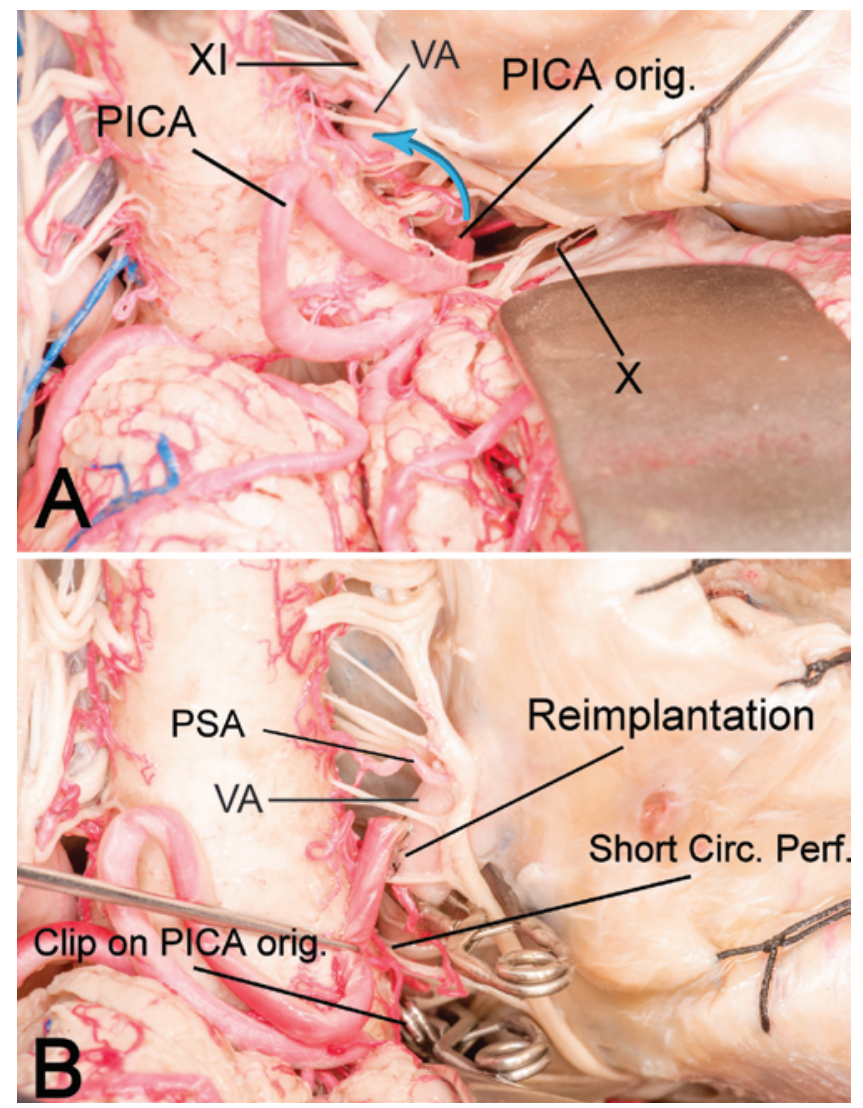

FIG. 2. Along-VA mobilization of the PICA (paradigm A). A: Far-lateral exposure of the left cerebellomedullary angle. One short circumflex perforator originates from the $P_{1}$ segment. The curved blue arrow shows the planned mobilization of the PICA. B: The origin of the PICA is clipped and cut, and the artery is mobilized proximally along the VA and reimplanted. Two other clips are placed on the presumed aneurysmal segment of the VA. No tension was imposed on the perforators or the PICA. Circ. = circumflex; orig. = origin; Perf. = perforator; PSA = posterior spinal artery; $X=10$ th cranial nerve; $X I=11$ th cranial nerve. Figure is available in color online only.

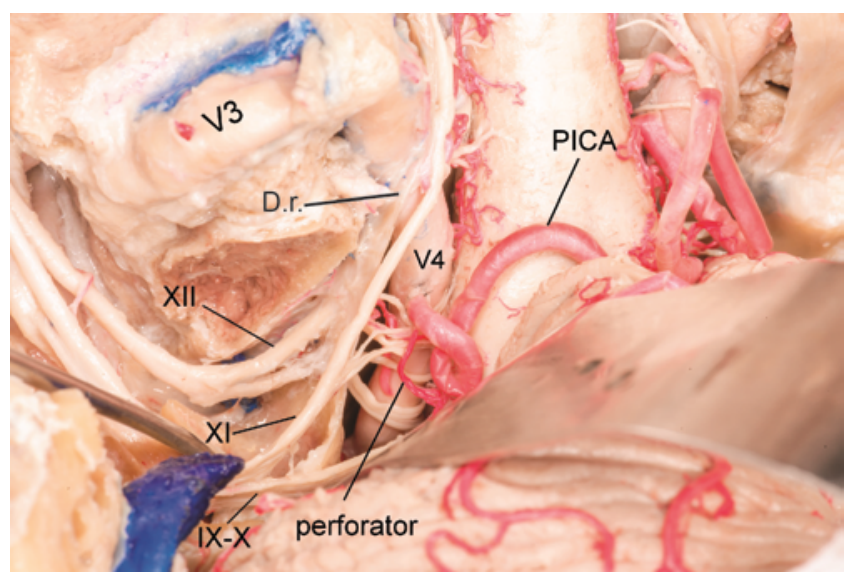

FIG. 3. Across-VA mobilization of the PICA (paradigm B). Far-lateral exposure of the right cerebellomedullary angle. After excising the available buffer length of the PICA, it is reimplanted on its origination site respecting its perforator branch. D.r. = dural ring; $I X=$ ninth cranial nerve; $X=10$ th cranial nerve; $X I=11$ th cranial nerve; $X I I=12$ th cranial nerve. Figure is available in color online only.

the PICA along the axis of the VA was $13.43 \mathrm{~mm}$ (range 5-22 mm; SD 4.61) and the average buffer length perpendicularly across the VA was $6.97 \mathrm{~mm}$ (range 0-16.4 $\mathrm{mm}$; SD 4.04). No strong correlation was found between $\mathrm{P}_{1}$ or $\mathrm{P}_{2}$ lengths and either of the buffer lengths measured. Similarly, no correlation was identified when the sum of $\mathrm{P}_{1}$ and $\mathrm{P}_{2}$ length was used. When the PICAs without $\mathrm{P}_{1}$ segments were excluded, the mean buffer length was increased along-VA $(14.57 \mathrm{~mm})$, whereas it was decreased across-VA $(6.88 \mathrm{~mm})$; these differences were not significant $(\mathrm{p}>0.05)$.

\section{Discussion}

Brainstem perforators and the buffer lengths of the PICA are the major features that determine the mobility of the recipient artery and feasibility of PICA reimplantation. The present study provides information about the anatomy of the proximal PICA that impacts the feasibility of PICA reimplantation in 2 clinical settings encountered regularly in practice-namely, the fusiform VA dissection and the nonclippable, proximal PICA aneurysm. Studies by Durward and Hamada et al. each reported PICA reimplantation in the case of VA dissection. ${ }^{8,12}$ They emphasized the need to mobilize the artery "in a gentle fashion" to prevent overtension of the brainstem perforators. ${ }^{8,12}$ However, the impact of the branching pattern of the perforators on the feasibility of PICA reimplantation has not been addressed in previous reports..$^{8-10,12,22,23}$

Brainstem-perforating arteries branching from the PICA originate from the medullary segments (i.e., $\mathrm{P}_{1}, \mathrm{P}_{2}$, and $\left.\mathrm{P}_{3}\right) \cdot{ }^{18}$ Lister et al. have subdivided these perforators into 3 types: direct perforators that course straight to the brainstem and short and long circumflex perforators that travel $<90^{\circ}$ and $>90^{\circ}$ around the brainstem before entering the parenchyma, respectively. ${ }^{18}$ According to Lister et al., $\mathrm{P}_{1}$ gives rise to an average of 1 perforator (the most common short circumflex type), $\mathrm{P}_{2}$ gives rise to an average of 1.8 perforators, and $\mathrm{P}_{3}$ has the most perforators (an 
TABLE 1. Characteristics of the anterior and lateral medullary segments and buffer lengths of the PICAs examined in the present study

\begin{tabular}{|c|c|c|c|c|c|c|c|c|c|c|}
\hline Specimen & $\mathrm{P}_{1}$ Length $(\mathrm{mm})$ & Direct & Short Circ & Long Circ & $\mathrm{P}_{2}$ Length $(\mathrm{mm})$ & Direct & Short Circ & Long Circ & $\begin{array}{c}\text { Along-VA } \\
\text { Buffer (mm) }\end{array}$ & $\begin{array}{c}\text { Across-VA } \\
\text { Buffer (mm) }\end{array}$ \\
\hline 1 & 0 & 0 & 0 & 0 & 7 & 0 & 0 & 0 & 5 & 10 \\
\hline 2 & 14 & 0 & 0 & 1 & 21 & 0 & 1 & 0 & 7 & 9 \\
\hline 3 & 4 & 0 & 0 & 0 & 10 & 1 & 0 & 0 & 15.7 & 7.5 \\
\hline 4 & 4 & 0 & 0 & 0 & 6 & 0 & 0 & 0 & 12.5 & 6 \\
\hline 5 & 15 & 0 & 2 & 0 & 10 & 0 & 2 & 0 & 22 & 10 \\
\hline 6 & 0 & 0 & 0 & 0 & 7.1 & 0 & 1 & 0 & 7.6 & 1 \\
\hline 7 & 0 & 0 & 0 & 0 & 8 & 0 & 0 & 0 & 12.1 & 6 \\
\hline 8 & 7.5 & 0 & 0 & 1 & 7.8 & 1 & 0 & 0 & 16.4 & 2 \\
\hline 9 & 11.8 & 0 & 1 & 0 & 9.9 & 0 & 1 & 0 & 16.3 & 4.5 \\
\hline 10 & 12.5 & 0 & 0 & 0 & 4 & 0 & 0 & 1 & 9 & 11 \\
\hline 11 & 16.5 & 0 & 1 & 1 & 14 & 0 & 0 & 0 & 10.3 & 9 \\
\hline 12 & 4.5 & 0 & 0 & 0 & 5.3 & 0 & 0 & 0 & 11.9 & 6.8 \\
\hline 13 & 9.8 & 0 & 1 & 0 & 5.2 & 0 & 1 & 0 & 15.1 & $0^{*}$ \\
\hline 14 & 6 & 0 & 1 & 0 & 5.6 & 0 & 1 & 0 & 17 & 3 \\
\hline 15 & 11.4 & 0 & 0 & 0 & 7.3 & 0 & 1 & 0 & 19.5 & 16.4 \\
\hline $16 \dagger$ & 0 & 0 & 0 & 0 & 0 & 0 & 0 & 0 & 14.2 & 3 \\
\hline 17 & 3.2 & 0 & 0 & 0 & 7.7 & 0 & 1 & 0 & 16.8 & 4.3 \\
\hline Mean \pm SD & $7.07 \pm 5.7$ & & NA & & $8.05 \pm 4.5$ & & NA & & $13.43 \pm 4.61$ & $6.97 \pm 4.04$ \\
\hline
\end{tabular}

Circ = circumflex; $\mathrm{NA}=$ not applicable.

* In this artery, early branching precluded any mobilization according to the across-VA paradigm.

$\dagger$ The PICA originated proximally from the VA without a $P_{1}$ or $P_{2}$ segment but was still mobile enough to give rise to extra length for reimplantation.

average of 3.3). They reported that the short circumflex perforator was the most common perforating type stemming from $\mathrm{P}_{1}$ and $\mathrm{P}_{2}$ segments. Shrontz et al. stated that more brainstem perforators arise from the distal cisternal portion of the PICA $\left(\mathrm{P}_{2}\right.$ and $\left.\mathrm{P}_{3}\right)$ than the proximal cisternal segment $\left(\mathrm{P}_{1}\right)$; these authors considered the proximal cisternal segment to be the most suitable site to perform anastomosis. ${ }^{24}$

In the present study, the least common perforator type in both $\mathrm{P}_{1}$ and $\mathrm{P}_{2}$ was the direct type (only 2 direct perforators from all $\mathrm{P}_{2}$ segments examined and no direct perforators from $\mathrm{P}_{1}$ ). This finding is different from that of Lister et al., who reported that the long circumflex type was the least common perforating type. ${ }^{18}$ Such a discrepancy may be due to our strict criteria for categorizing direct perforators. To avoid ambiguity, we designated a perforator as direct only when it was directly coursing toward the adjacent brainstem, without coursing around the brainstem. Using the criteria defined by Lister et al., a short circumflex perforator that travels a very short distance (e.g., < $10^{\circ}$ ) around the brainstem before entering the parenchyma may be easily mistaken for a direct perforator and vice versa.

\section{Reimplantation Along the VA}

Good mobility of the PICA along the VA (mean buffer length $13.43 \mathrm{~mm}$ ) resulted from an absence of direct perforating branches in the $\mathrm{P}_{1}$ segment and few short circumflex perforators in the $\mathrm{P}_{1}$ and $\mathrm{P}_{2}$ segments. Although we did not measure perforator lengths, the mobility of the PICA along the VA is related to the length of perforating arteries originating from the mobilized segments (i.e., $\mathrm{P}_{1}$ and $\mathrm{P}_{2}$ ). We did not mobilize the $\mathrm{P}_{3}$ segment due to its numerous direct perforating branches to the brainstem. However, in 1 exception (Specimen 14; Table 1), without $\mathrm{P}_{1}$ and $\mathrm{P}_{2}$ segments and with a relatively lengthy $\mathrm{P}_{3}$ segment, mobilization of the cisternal portion of the PICA was performed without tension to the perforators. A direct perforating artery from $\mathrm{P}_{1}$ tethers the PICA to the medulla, and any mobilization of the PICA along the brainstem is limited. On the other hand, even a short PICA can give rise to a sizable buffer length for reimplantation along the VA, because a circumflex perforator will allow movement of the first segment. In the majority of the PICAs we studied, the perforating branches from the first 2 segments of the artery were circumflex types (Fig. 2B).

The results of our study may provide important information to guide preoperative decision making. Dissecting VA aneurysms are an average of $11.1 \pm 6.5 \mathrm{~mm}$ in length. ${ }^{6}$ The maximum distance required for a PICA to be transposed along a VA (aneurysmal) is nearly half the length of the aneurysm. Considering the buffer length found in this study $(13.43 \pm 4.61 \mathrm{~mm})$, most dissecting intradural VA aneurysms are amenable to PICA reimplantation after trapping the aneurysm. Of note, a nondiseased segment of the VA should be chosen for reimplantation, ${ }^{5}$ a property that is verified only after the arterial wall is inspected intraoperatively. Perforator anatomy is not typically visualized preoperatively on angiography; therefore, limitations related to direct perforators must also be determined intraoperatively. Also, if the reimplantation site is selected distal to the aneurysm, depth of the operative field may add complexity to the technique and must be considered before attempting the reimplantation. 


\section{Reimplantation Across the VA}

The mean buffer length available for reimplantation across the VA was $6.97 \mathrm{~mm}$, which is substantially shorter than the buffer length measured for reimplantation along the VA. Therefore, mobilization of the PICA across the VA is more limited than mobilization along the VA (Fig. 3). This difference is due to: 1) the distance between the origin of the PICA and the origin of the first perforating branch, and 2) the length of the perforating branches along the cisternal segment of the artery. The former parameter limits the absolute available buffer length available for excision and the latter controls the mobility of the PICA from its primary position. However, if the first perforating branch originates early along the course of the PICA (the first factor), the available buffer length of the PICA will be greatly limited. Although we did not measure the distance from the origin of the PICA to the origin of the first perforating branch, such a limiting configuration occurred in $5(29 \%)$ of our specimens. In the remainder, a minimum buffer length of $4.5 \mathrm{~mm}$ was available (Table 1).

Aneurysms of the proximal PICA range greatly in size ${ }^{21}$ with a mean diameter $<10 \mathrm{~mm}$ and most of them located at the VA-PICA junction. ${ }^{13,16,21,26}$ This pathology makes PICA reimplantation a viable option when there is a nonclippable, proximal PICA aneurysm that measures $<6.9 \mathrm{~mm}$ in length (the mean across-VA buffer length). However, nonclippable PICA aneurysms $>6.9 \mathrm{~mm}$ may be optimally treated using other revascularization procedures, e.g., a side-to-side anastomosis to the contralateral PICA.

Several reports have described reconstruction of the PICA after resection of its proximal portion, ${ }^{11,12}$ but the resected length of the PICA was not stated. For example, Hamada et al. used the superficial temporal artery as an interposition graft to reconstruct the PICA in a case of VA aneurysm with an adherent proximal PICA along the wall of the aneurysm. ${ }^{11}$ The results of our study may provide important information about the feasibility of a reimplantation without an interposed graft or extracranial-intracranial bypass.

The use of preoperative angiographic studies may help neurosurgeons decide whether a nonclippable, proximal PICA aneurysm is amenable to trapping and reimplantation. But again, preoperative angiography may not visualize proximal perforator anatomy. Our results should be considered as a rough guide. The reimplantation technique must be carefully applied only after considering the specific anatomy of the aneurysm, its relationship to perforators and cranial nerves, the technical difficulty of the anastomosis, and the feasibility of easier options.

\section{Conclusions}

Although PICA reimplantation is a less common technique of revascularization in the PICA territory, ${ }^{19}$ it can be considered in certain cases where other options (e.g., PICA-PICA bypass, excision-reanastomosis, or occipital artery-to-PICA anastomosis) are not favorable. ${ }^{11,19}$ This revascularization technique maintains the original vascular pattern and avoids harvesting distant donor arteries. However, PICA reimplantation is not always a good choice; the anatomical limitations imposed by perforators and the condition of the VA wall ${ }^{5,8}$ must be taken into account. The PICA anatomy and buffer lengths measured in this study might help guide the choice of revascularization technique used with PICA aneurysms and further clarify the role of this reimplantation technique in the current practice of cerebrovascular surgery.

\section{Acknowledgments}

We thank Mahnaz Zamani for her outstanding contribution to the artwork in this article. We also express our gratitude to the body donors and their families, who through their altruism contributed to making this project possible.

\section{References}

1. Ali MJ, Bendok BR, Tawk RG, Getch CC, Batjer HH: Trapping and revascularization for a dissecting aneurysm of the proximal posteroinferior cerebellar artery: technical case report and review of the literature. Neurosurgery 51:258-263, 2002

2. Bacigaluppi S, Bergui M, Crobeddu E, Garbossa D, Ducati A, Fontanella M: Aneurysms of the medullary segments of the posterior-inferior cerebellar artery: considerations on treatment strategy and clinical outcome. Neurol Sci 34:529536, 2013

3. Benet A, Prevedello DM, Carrau RL, Rincon-Torroella J, Fernandez-Miranda JC, Prats-Galino A, et al: Comparative analysis of the transcranial "far lateral" and endoscopic endonasal "far medial" approaches: surgical anatomy and clinical illustration. World Neurosurg 81:385-396, 2014

4. Benet A, Rincon-Torroella J, Lawton MT, González Sánchez $\mathrm{JJ}$ : Novel embalming solution for neurosurgical simulation in cadavers. J Neurosurg 120:1229-1237, 2014

5. Czabanka M, Ali M, Schmiedek P, Vajkoczy P, Lawton MT: Vertebral artery-posterior inferior cerebellar artery bypass using a radial artery graft for hemorrhagic dissecting vertebral artery aneurysms: surgical technique and report of 2 cases. J Neurosurg 114:1074-1079, 2011

6. Deng D, Jin D, Zhou J, Chang Q, Qu K: Characteristics and endovascular treatment of intracranial vertebral artery aneurysms. Neurol India 59:833-838, 2011

7. Dolenc V: End-to-end suture of the posterior inferior cerebellar artery after the excision of a large aneurysm: case report. Neurosurgery 11:690-693, 1982

8. Durward QJ: Treatment of vertebral artery dissecting aneurysm by aneurysm trapping and posterior inferior cerebellar artery reimplantation. Case report. J Neurosurg 82:137-139, 1995

9. Evans JJ, Sekhar LN, Rak R, Stimac D: Bypass grafting and revascularization in the management of posterior circulation aneurysms. Neurosurgery 55:1036-1049, 2004

10. Guo L, Wan J, Qiu Y, Zhang X: Management of hemorrhagic dissecting vertebral artery aneurysms involving posterior inferior artery. J Craniofac Surg 25:674-676, 2014

11. Hamada J, Nagahiro S, Mimata C, Kaku T, Ushio Y: Reconstruction of the posterior inferior cerebellar artery in the treatment of giant aneurysms. Report of two cases. J Neurosurg 85:496-499, 1996

12. Hamada J, Todaka T, Yano S, Kai Y, Morioka M, Ushio Y: Vertebral artery-posterior inferior cerebellar artery bypass with a superficial temporal artery graft to treat aneurysms involving the posterior inferior cerebellar artery. J Neurosurg 96:867-871, 2002

13. Horowitz M, Kopitnik T, Landreneau F, Krummerman J, Batjer HH, Thomas G, et al: Posteroinferior cerebellar artery aneurysms: surgical results for 38 patients. Neurosurgery 43:1026-1032, 1998 
14. Hudgins RJ, Day AL, Quisling RG, Rhoton AL Jr, Sypert GW, Garcia-Bengochea F: Aneurysms of the posterior inferior cerebellar artery. A clinical and anatomical analysis. J Neurosurg 58:381-387, 1983

15. Kanou Y, Arita K, Kurisu K, Ikawa F, Eguchi K, Monden S, et al: Dissecting aneurysm of the peripheral posterior inferior cerebellar artery. Acta Neurochir (Wien) 142:1151-1156, 2000

16. Kleinpeter G: Why are aneurysms of the posterior inferior cerebellar artery so unique? Clinical experience and review of the literature. Minim Invasive Neurosurg 47:93-101, 2004

17. Lehto H, Harati A, Niemelä M, Dashti R, Laakso A, Elsharkawy A, et al: Distal posterior inferior cerebellar artery aneurysms: clinical features and outcome of 80 patients. World Neurosurg 82:702-713, 2014

18. Lister JR, Rhoton AL Jr, Matsushima T, Peace DA: Microsurgical anatomy of the posterior inferior cerebellar artery. Neurosurgery 10:170-199, 1982

19. O'Shaughnessy BA, Getch CC, Bowman RM, Batjer HH: Ruptured traumatic vertebral artery pseudoaneurysm in a child treated with trapping and posterior inferior cerebellar artery reimplantation. Case report and review of the literature. J Neurosurg 102 (2 Suppl):231-237, 2005

20. Rodríguez-Hernández A, Rhoton AL Jr, Lawton MT: Segmental anatomy of cerebellar arteries: a proposed nomenclature. Laboratory investigation. J Neurosurg 115:387-397, 2011

21. Salcman M, Rigamonti D, Numaguchi Y, Sadato N: Aneurysms of the posterior inferior cerebellar artery-vertebral artery complex: variations on a theme. Neurosurgery 27:1221, 1990

22. Sanai N, Zador Z, Lawton MT: Bypass surgery for complex brain aneurysms: an assessment of intracranial-intracranial bypass. Neurosurgery 65:670-683, 2009

23. Shin YS, Kim SY, Cho KH, Cho KG: Treatment of vertebral artery dissecting aneurysms presenting with progressive myelopathy. J Clin Neurosci 11:896-898, 2004

24. Shrontz C, Dujovny M, Ausman JI, Diaz FG, Pearce JE, Berman SK, et al: Surgical anatomy of the arteries of the posterior fossa. J Neurosurg 65:540-544, 1986

25. Welch BG, Batjer HH: Distal aneurysms of the posterior inferior cerebellar artery: the rare surgical lesion. World Neurosurg 83:466-467, 2015

26. Wu J, Xu F, Yu ZQ, Zhou YX, Cui G, Li XD, et al: Clinical experiences of ruptured posteroinferior cerebellar artery aneurysms and anatomical analysis in the cadaver in a single center of China. Clin Neurol Neurosurg 114:366-371, 2012

\section{Disclosures}

The authors report no conflict of interest concerning the materials or methods used in this study or the findings specified in this paper.

\section{Author Contributions}

Conception and design: Benet, Tayebi Meybodi, Lawton. Acquisition of data: Tayebi Meybodi, Feng. Analysis and interpretation of data: Benet, Tayebi Meybodi. Drafting the article: Benet, Tayebi Meybodi, Feng. Critically revising the article: all authors. Reviewed submitted version of manuscript: Benet, Lawton, Feng. Approved the final version of the manuscript on behalf of all authors: Benet. Statistical analysis: Benet, Tayebi Meybodi. Administrative/technical/material support: Benet, Lawton. Study supervision: Benet, Lawton, Feng.

\section{Correspondence}

Arnau Benet, Department of Neurological Surgery, University of California, San Francisco, 505 Parnassus Ave., M-779, San Francisco, CA 94143. email: arnaubenet@gmail.com. 